\title{
Magnitude estimation and sensory matching
}

\author{
LAWRENCE E. MARKS \\ John B. Pierce Foundation Laboratory and Yale University, New Haven, Connecticut
}

\begin{abstract}
Scaling procedures are commonly used to generate sensory matches, in which each match is defined as the set of stimuli that yield a constant average judgment. Five experiments evaluated the principle of "matching-by-scaling" in the magnitude estimation of loudness: (1) Matches derived from magnitude estimates of the loudness of qualitatively different sounds $(500-\mathrm{Hz}$ and $2500-\mathrm{Hz}$ tones) change dramatically with changes in the relative intensity levels (contexts) of the stimuli being judged. (2) Small changes in matches apparently can arise from sequential processes of judgment, even when the stimuli are qualitatively identical (all $500 \mathrm{~Hz}$ ). (3) Even when the effects of sequential processes are minimized by averaging, changing the context induces large changes in loudness matches derived from magnitude estimates of $500-\mathrm{Hz}$ and 2500 . $\mathrm{Hz}$ tones. (4) Changing contextual sets of qualitatively identical stimuli (all $500 \mathrm{~Hz}$ ) presented to different ears has little effect on interaurally matching values. (5) Matches between $500-\mathrm{Hz}$ and $2500-\mathrm{Hz}$ tones shift with changes in the proportions of presentations of various intensity levels, even when the intensity levels themselves do not change. The results imply that judgments of perceived intensity of qualitatively different stimuli do not always obey the principle that equal sensations yield equal response, and, accordingly, that experiments using scaling methods to generate matches should compare stimuli whose levels of subjective magnitude are roughly the same. The results can be described by a quantitative model containing a parameter that depends on qualitative similarity and that may therefore reflect the degree of overlap in activated sensory channels.
\end{abstract}

Central to the discipline of psychophysics is the determination of equality between two perceptual experiences, an operation known as matching. Matching may involve either perceptual identity, in which percepts are wholly indistinguishable, or it may involve perceptual equivalence, in which percepts are equal with respect to some attribute or attributes, but not with respect to others.

Brindley (1960) dubbed these two kinds of matching operations Class A and Class B, respectively. Metameric color matching is a prototypical Class $\mathrm{A}$ operation (metamers are lights of different spectral composition that appear equal in all aspects-hue, saturation, and brightness). Heterochromatic brightness matching and heterofrequency loudness matching exemplify Class B operations: Lights may be equal in brightness but different in color; sounds may be equal in loudness but different in pitch. Much of the edifice of sensory psychophysics rests on these types of matching.

My main concern here is the partial equivalence of matches established by Class B operations-in particular, intensity matches in the face of qualitative differences. The notion of partial equivalence relies implicitly on the reasonable view that for any attribute or set of attributes, there do exist absolute equations across stimuli that differ in physical composition. For instance, under fixed con-

This research was supported by NSF Grant BNS 83-16098 and NIH Grant NS21326. I thank Elizabeth Warner for her assistance with the experiments, and Joseph Stevens and Barry Green for their comments on an early version of this manuscript. Address requests for reprints to Lawrence E. Marks, John B. Pierce Foundation Laboratory, 290 Congress Avenue, New Haven, CT 06519. ditions of stimulation and state of the sense organ, and within limits set by precision of psychophysical methods, a given subject will perceive a $500-\mathrm{Hz}$ tone at $70 \mathrm{~dB}$ SPL and $2500-\mathrm{Hz}$ tone at some other SPL to be equally loud. Of course, in any particular experimental implementation, it is necessary to avoid certain methodological biases of matching in order to ensure adequately precise, reliable, and valid measurement. But such biases are seen as largely of the second order.

Scaling methods such as magnitude estimation currently find wide use in sensory psychophysics-and elsewhereas a means, albeit indirect, to assess matching performance. When used for this purpose, magnitude estimation asks subjects to assign numbers to represent the perceived intensities of stimuli that differ in perceived quality or in other dimensions besides intensity; that is, subjects make their judgments under conditions corresponding to Class B matching. Prima facie, it would appear that magnitude estimates can specify-quickly, efficiently, and reliably-equivalences across qualitatively different stimuli.

Several investigators, including the present author, have reported how magnitude estimates produce the same sensory equalities as those produced by direct matching. J. C. Stevens and S. S. Stevens (1963), for instance, investigated the perception of brightness as a function of the level of light adaptation that operated just before brief visual stimulation; they found that magnitude estimation and interocular matching yielded equivalent matches between luminance levels presented to a dark-adapted eye and to a light-adapted eye. Hellman and Zwislocki (1964) 
found that magnitude scaling and direct matching yielded equivalent loudness matches between $1000-\mathrm{Hz}$ tones heard in quiet and in the presence of masking noise. More than a decade ago, I documented and reviewed a wide range of experiments that made the general point (Marks, 1974): Scaling procedures, such as magnitude estimation, can function as "null methods," quite apart from their putative ability to represent sensory magnitudes on a ratio scale.

My aim here is to evaluate more critically than has generally been done in the past the principle of "matchingby-scaling," that is, the principle that scaling methods always produce sensory matches when subjects judge the intensity of qualitatively different stimuli. The principle rests on the seemingly reasonable assumption that subjects will make the same overt response, on average, whenever the sensory-perceptual experience has a particular value on the dimension being judged. In judging the loudness of pure tones differing in frequency, for example, subjects presumably give the same rating-the same average magnitude estimation or whatever-to the same loudness, regardless of sound frequency. But is this true? Some recent evidence suggested to me that there may be unexpected limits to the validity of the matchingby-scaling principle and thus provided the impetus for the experiments described below.

My concerns come from two sources. First is the finding that cross-modal matches change when the relative intensity levels (contexts) of the stimuli change (Marks, Szczesiul, \& Ohlott, 1986): Two scaling methods, magnitude estimation and cross-modal difference estimation, agreed in showing that cross-modal matches between loudness and vibration, between loudness and brightness, and between auditory duration and visual duration were all markedly influenced by the contextual sets of stimuli being compared. Simply put, when subjects compared a fixed set of stimulus levels on one modality to a variable set on the other, the matching values changed with changes in the stimulus levels of the variable set. With auditory duration and visual duration, the changes in matching values were about one-third the size of the changes in stimulus levels; with loudness and vibration and with loudness and brightness, the changes in matching values were about half the size of the changes in stimulus context (although I should note that J. C. Stevens \& Marks, 1980, reported much smaller effects of changing stimulus context on derived loudness-brightness matches). This outcome indicates a substantial relativistic component to cross-modal matching. Several investigators have argued that cross-modal matches are not absolute but relative, that they depend primarily on context (Cohen, 1934; Mellers \& Birnbaum, 1982; see also K. Smith \& Hardy, 1961). Moreover, the large relativistic component to the judgment of duration as well as intensity suggests that such phenomena may be widespread.

Relativity in cross-modal judgment need not, of course, imply relativity in intramodal judgment, and it is intramodal judgment that forms the subject of the present study. The second impetus for the present study came from my recent attempts (not published) to use the method of magnitude estimation to assess loudness summation in individual subjects. Those experiments compared single tones to two-tone complexes comprising equally loud, widely separated sound frequencies-a condition in which I expected the two-tone complexes to be, on average, twice as loud as the single tones (e.g., Fletcher \& Munson, 1933; Howes, 1950; Marks, 1979). Instead, I found that repeated presentation of the stimuli yielded less than complete summation - as if, with multiple replications of the stimulus sets, there was a contextual "assimilation" of the judgments. In other words, these unexpected findings suggest shifts in loudness matches between single tones and multiple tones. The dependence of loudness matches on stimulus context became the subject of the present study, which found that marked relativity can characterize intramodal matches derived from scaling judgments.

\section{EXPERIMENT 1}

The first experiment provided a simple and direct test of magnitude estimation as a means to generate sensory matches. Despite the positive successes of Hellman and Zwislocki (1964), of J. C. Stevens and S. S. Stevens (1963), and of others outlined above, the possibility remains that under certain circumstances, matches derived from scaling judgments can be strongly biased. I suspect, given the findings on relativity in cross-modal comparisons (Marks et al., 1986), that such biasing will occur-or less pejoratively, that matches will vary-when two conditions are satisfied: First, the stimuli being "matched" must be qualitatively dissimilar, and second, the sets of qualitatively different stimuli being judged must have different levels or ranges of subjective intensities.

Let me take up these two conditions in order. First, in cross-modal comparison, the stimuli being matched are, by their very nature, qualitatively different. Presumably, stimuli from two modalities, such as a white light and a pure tone, are even more different than stimuli from the same modality, such as pure tones of different frequencies. Nevertheless, qualitatively different homomodal stimuli may behave much like qualitatively different heteromodal stimuli. Second, the cross-modally matching values change when the stimulus context changes, that is, when the psychological magnitudes of the stimuli being compared are shifted relative to each other (Marks et al., 1986). In cross-modal comparison, changing the levels of sound intensity, while leaving intact the levels of vibration or light intensity, resulted in a substantial shift in the matching stimulus levels. This paradigm tests the stability of matches when the entire psychophysical system is "challenged."

Experiment 1 put intramodal comparison to the same test: In different experimental sessions, subjects judged the loudness of various levels of pure tones of 500 and $2500 \mathrm{~Hz}$. In one session, the sound-pressure levels (SPLs) 
at $500 \mathrm{~Hz}$ were low, and those at $2500 \mathrm{~Hz}$ were high; in the other, the relative levels were reversed. The question is, Do matching values remain invariant across shifts in stimulus context?

\section{Method}

The stimuli consisted of $1-\mathrm{sec}$ bursts of $500-\mathrm{Hz}$ and $2500-\mathrm{Hz}$ tones, with $10-\mathrm{msec}$ rise and decay, presented binaurally through TDH-39 headphones mounted in MX41/AR cushions. The stimuli were generated by Coulbourn Instruments modules under the control of an Apple IIe microcomputer. In one condition (A), the SPLs of the 500-Hz signals were low (35-70 dB, in 5-dB steps) and those of the $2500-\mathrm{Hz}$ signals were high $(50-85 \mathrm{~dB}$, in $5-\mathrm{dB}$ steps); in the other condition (B), the SPLs of the $500-\mathrm{Hz}$ signals were high (55-90 dB) and those of the $2500-\mathrm{Hz}$ signals were low (30-65 dB).

Thus, at each frequency, the SPLs differed across the two conditions by $20 \mathrm{~dB}$, and because the sets shifted in opposite directions, the overall contextual shift between the two sets of stimuli was $40 \mathrm{~dB}$. The use of slightly greater SPLs at $500 \mathrm{~Hz}$ than at $2500 \mathrm{~Hz}$ represented an attempt to equalize loudness at the two frequencies, given the auditory system's greater sensitivity at $2500 \mathrm{~Hz}$. Note that this selection of stimulus values reflects the view that there do exist absolute equivalences between loudnesses at different sound frequencies.

The method was magnitude estimation with no specified modulus. The subjects were instructed to assign to the first stimulus whatever number they deemed appropriate to stand for its loudness, and then to assign to succeeding stimuli other numbers in proportion. Whole numbers, decimals, and fractions were permitted. The numeral "zero" reflected the absence of sound, but it was never reported. From each trial to the next, the two frequencies were presented in strict alternation-the procedure used by $\mathrm{J}$. C.
Stevens and Marks (1980) and by Marks et al. (1986) in their assessments of the "absoluteness" of cross-modal matches. Otherwise, the order of stimulus levels was random and different in every session for every subject. The entire set of 16 stimuli (8 at each frequency) was presented and judged a total of 10 times within the course of each session by each of the 16 young men and women who served as subjects. Each subject served in two such sessions, one judging the stimuli of Set $A$, the other judging those of Set $B$. Eight of the subjects participated first in Condition $A$ and then in Condition B; the sequence was reversed for the other 8 subjects. Sessions were separated by 1-10 days.

\section{Results and Discussion}

The magnitude estimates given by each subject to each stimulus were averaged arithmetically within sessions, and these subject means were then averaged geometrically across subjects in each condition (see Figure 1). Inspection of Figure 1 makes it clear that the contextual set of stimuli exerted a substantial effect on the relative judgments of loudness at the two frequencies. Although the slopes of the functions did not change over conditions, the intercepts changed markedly at both sound frequencies (a bit more, Figure 1 indicates, at $2500 \mathrm{~Hz}$ than at $500 \mathrm{~Hz}$ ). And because intercepts changed, matching values changed: When the SPLs at $500 \mathrm{~Hz}$ were low (open squares) and those at $2500 \mathrm{~Hz}$ were high (open triangles), the $500-\mathrm{Hz}$ signals yielded greater loudness judgments at equally intense levels. But when the SPLs at $500 \mathrm{~Hz}$ were high (filled squares) and those at $2500 \mathrm{~Hz}$ were low (filled triangles), just the reverse occurred, with the $2500-\mathrm{Hz}$

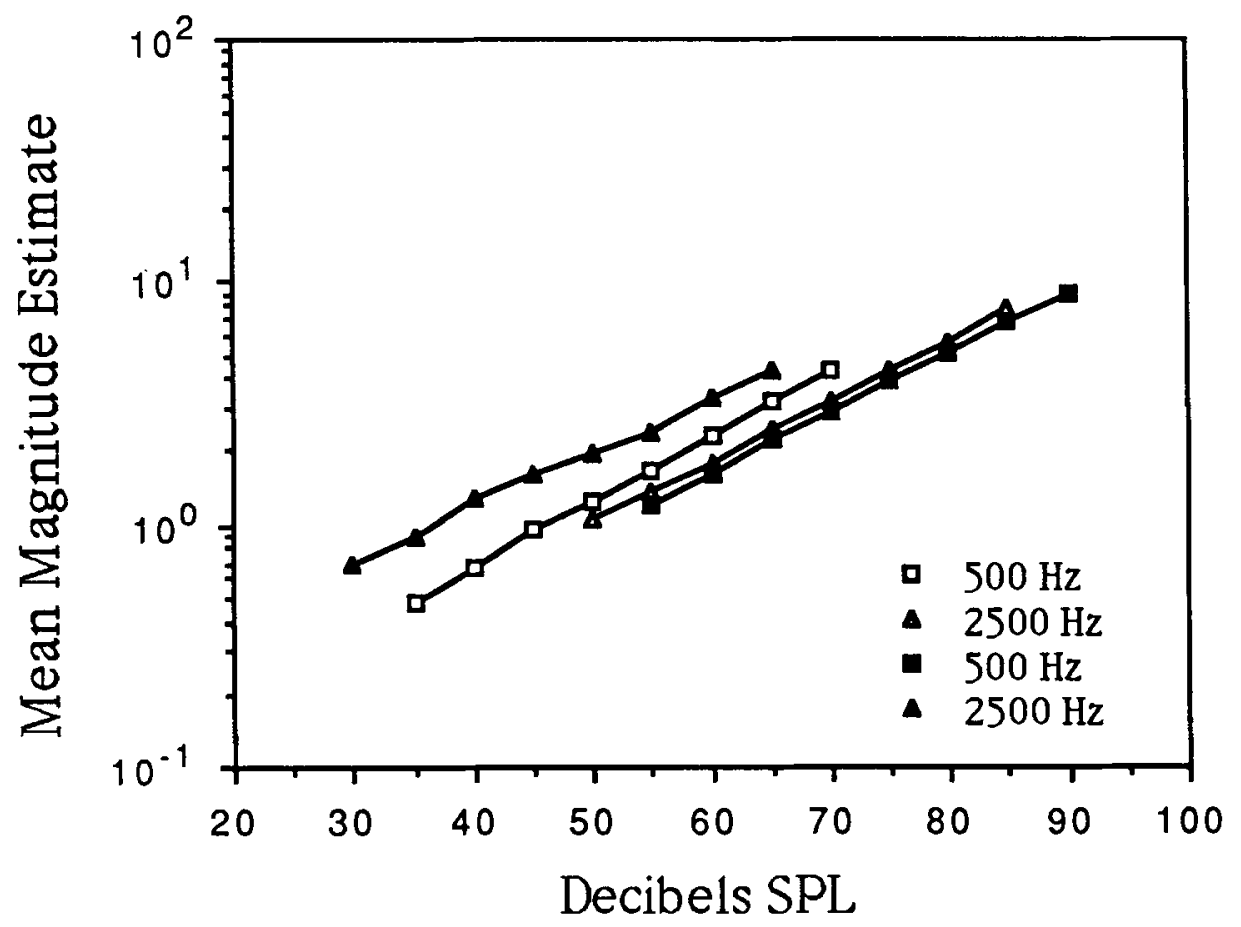

Figure 1. Geometric mean magnitude estimates of the loudness of $500-\mathrm{Hz}$ tones (squares) and 2500-Hz tones (triangles), plotted against sound-pressure level (SPL). In Condition A, the stimulus presentations alternated between low SPLs at $500 \mathrm{~Hz}$ and high SPLs at $2500 \mathrm{~Hz}$ (open symbols), and in Condition B, between high SPLs at $500 \mathrm{~Hz}$ and low SPLs at $2500 \mathrm{~Hz}$ (filled symbols). 


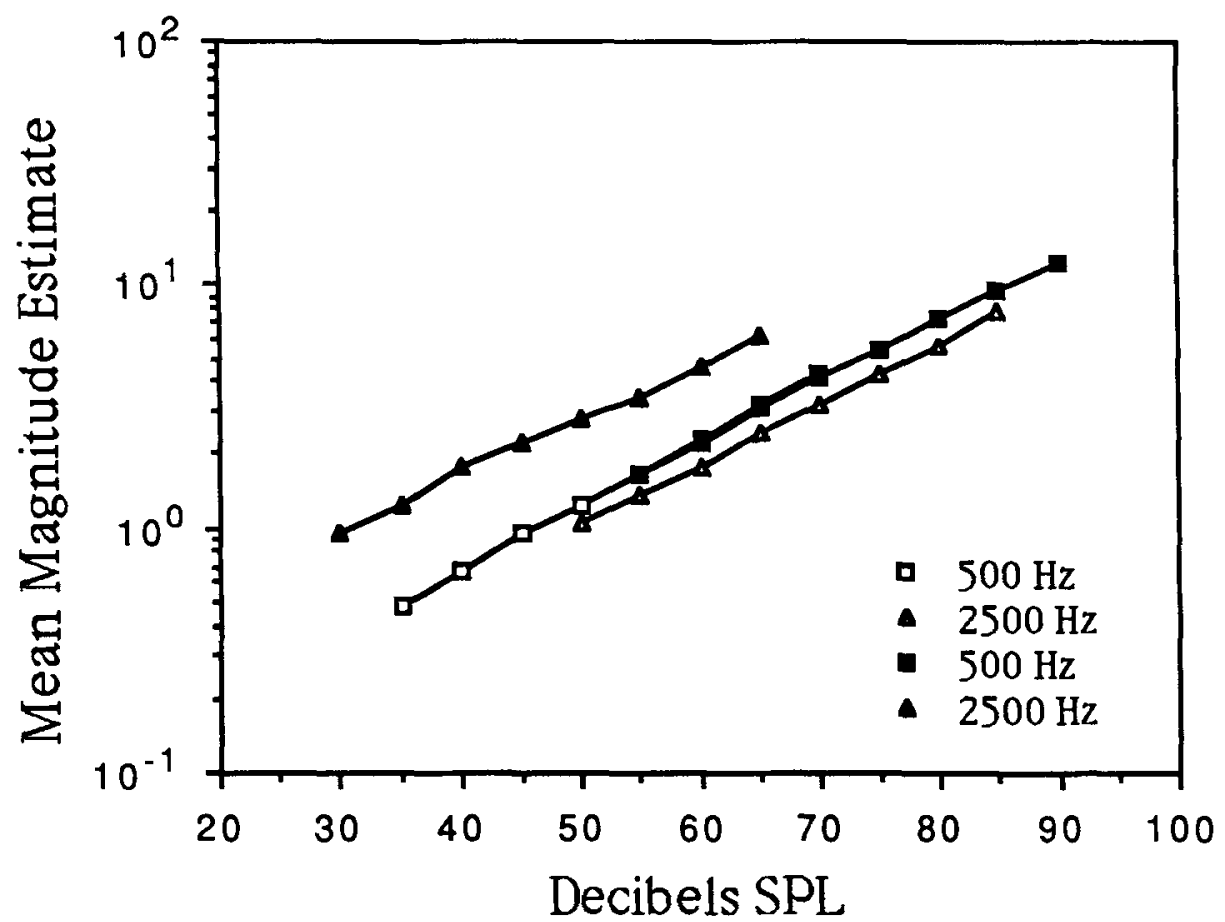

Figure 2. The data from Figure 1, replotted with the functions normalized. Normalization involved (1) shifting in the vertical plane the function at $500 \mathrm{~Hz}$ from Condition B (filled squares) so as to maximize its correspondence with the function at $500 \mathrm{~Hz}$ from Condition $A$ (open squares) (indicated by the virtual identity of the points from 55 to $70 \mathrm{~dB}$ ); and (2) then shifting by the same amount the function at $2500 \mathrm{~Hz}$ from Condition B (filled triangles). The separation of the functions at $2500 \mathrm{~Hz}$ now reveals the magnitude of the contextually induced shift in loudness matches.

signals yielding greater judgments. Exactly the same sort of changes appeared previously in cross-modal comparisons (Marks et al., 1986; see also Melamed, 1970); the changes in judgment induced by shifting relative stimulus levels may be characterized as "assimilative."

The magnitude of the difference between results for the two conditions is most readily assessed after the data are normalized. To do this, I calculated, for the four SPLs at $500 \mathrm{~Hz}$ common to Conditions $\mathrm{A}$ and $\mathrm{B}$, the ratio $F$ of the overall geometric mean from Set $A$ to that from Set $\mathrm{B}\left(\log F=[1 / \mathrm{n}] \Sigma \log R_{\mathrm{a} i} / R_{b i}\right)$, then multiplied all of the judgments of Set $B$, both at 500 and $2500 \mathrm{~Hz}$, by $F$. This transformation overlays the two functions at $500 \mathrm{~Hz}$ relating loudness judgment to SPL (Figure 2); consequently, the separation of the corresponding functions at $2500 \mathrm{~Hz}$ now represents the effect on judgment of shifting the contextual set. The transformation leaves intact, however, the implicit matching levels of loudness across sound frequency.

The context-induced change is approximately a $2: 1$ ratio in the loudness judgments themselves. However, following Marks et al. (1986), who pointed out that the change in such loudness ratios depends strongly on the ratio range of numbers assigned by the subjects, I also calculated the effect of context in terms of the change in loudness matches. Note that when the subjects judged the stimuli of Set A, the loudness of a $500-\mathrm{Hz}$ tone at $65 \mathrm{~dB}$ matched the loudness of a $2500-\mathrm{Hz}$ tone at $70 \mathrm{~dB}$ (both of those stimuli giving rise to the same mean magnitude estimate); but when the subjects judged the stimuli of Set B, the same $500-\mathrm{Hz}$ tone at $65 \mathrm{~dB}$ matched a 2500 $\mathrm{Hz}$ tone at $53 \mathrm{~dB}$. Thus the loudness match shifted by $17 \mathrm{~dB}$ !

The two functions at $2500 \mathrm{~Hz}$ (Figure 2) are separated overall by an average of $17.7 \mathrm{~dB}$, which thus represents the average change in matching values induced by shifting contextual sets (if we look at first sessions only, comparing the 8 subjects who judged Set $A$ to the 8 subjects who judged Set $B$, the difference is even larger$19.8 \mathrm{~dB}$ ). Given that the contextual shift between Stimulus Sets A and B amounted to $40 \mathrm{~dB}$ overall (a 20-dB shift at each frequency), a change of $17.7 \mathrm{~dB}$ in matches constitutes $44 \%$ of the contextual shift. This outcome is remarkable. Marks et al. (1986) reported percentage changes in cross-modal matches that were hardly larger (46\% between loudness and brightness and $56 \%$ between loudness and vibration). The present findings suggest that loudness matches obtained by scaling procedures like magnitude estimation can depend markedly on the choice of stimulus levels.

\section{EXPERIMENT 2}

It seems intuitively reasonable to expect that the bias exerted by context on matching values must depend at least in part on the degree of qualitative similarity between the 
stimuli being judged. In the limiting case, if the stimuli are identical, there should be no bias at all. At least, this should be the case if the contextually induced bias is not due to some other methodological characteristic of the experimental procedure, such as the strict alternation between stimulus frequencies from trial to trial.

But the procedure of alternating from trial to trial between stimuli selected from the two stimulus sets certainly could affect matches, given the evidence that sequential biases operate in judgment. The numerical response given to the current stimulus depends not only on such physical characteristics of that stimulus as its frequency and intensity, but also on prior stimuli and on responses to them. In general, sequential effects tend to contain two main components-contrast between the current response and the previous stimulus, but assimilation of the current response to the previous response (e.g., Jesteadt, Luce, \& Green, 1977; Lockhead \& King, 1983; Ward, 1982). Hence, a stimulus will tend to "mismatch" itself each time it follows a different prior stimulus or response, as Cross (1973, Figure 1), Lockhead and King (1983, Figure 2), and Ward (1973, Figure 2) have shown; overall, sequential effects tend to be largely assimilative, in that responses to current stimuli resemble responses to immediately prior stimuli.

Such sequential effects could influence matching values under the procedure of Experiment 1. In Condition A, the SPLs at $500 \mathrm{~Hz}$ were low and those at $2500 \mathrm{~Hz}$ were high, but in Condition B, the SPLs at $500 \mathrm{~Hz}$ were high and those at $2500 \mathrm{~Hz}$ were low. Because of the strict trialto-trial alternation between the two sound frequencies, each subset of stimuli would be subject to different sequential effects: In Condition A, the relatively soft 500$\mathrm{Hz}$ tones alternate with relatively loud $2500-\mathrm{Hz}$ tones. If sequential effects lead to assimilation, the loudness judgments of the $500-\mathrm{Hz}$ tones should increase, whereas those of the $2500-\mathrm{Hz}$ tones should decrease. Similarly, in Condition $\mathrm{B}$, the relatively loud $500-\mathrm{Hz}$ tones alternate with relatively soft $2500-\mathrm{Hz}$ tones, and, consequently, the loudness judgments of the former should decrease while those of the latter increase. Overall, then, one would expect different implicit loudness matches under the two conditions. Might these effects provide the entire explanation for the shift in matches observed in Experiment 1? Experiment 2 provided a simple empirical test, mimicking the conditions of the first experiment, except that in Experiment 2 the two sound frequencies were identical.

\section{Method}

As indicated, the only change from Experiment 1 was in the stimulus frequencies. Here all stimuli had a frequency of $500 \mathrm{~Hz}$. Nevertheless, the stimulus sets were treated as before: In Condition A, the SPLs of one subset were low $(35-70 \mathrm{~dB})$ and those of the other high $(55-90 \mathrm{~dB})$; in Condition $\mathrm{B}$, the subsets were reversed, the former now high and the latter low. Of course, from the subjects' point of view, the sets of stimuli in Conditions $A$ and B were absolutely identical, consisting in each case of SPLs of $35-90 \mathrm{~dB}$, with levels $55-70 \mathrm{~dB}$ common to the two subsets and thus presented twice as often as the lower and higher SPLs. But
}

the procedure does call for strict alternation from trial to trial between stimuli from the two subsets of each set; hence, in each session, the subjects were presented stimuli that alternated between low and high subsets of SPLs. Had the procedure involved complete randomization of stimulus levels, the present experiment would have been unnecessary, in that no difference could emerge between stimulus subsets (except that resulting from noise in the measurements). But this need not be the case with the present design.

Again, 16 young men and women served as subjects, each in two sessions, judging all 16 stımuli a total of 10 times in each session.

\section{Results and Discussion}

Context had only a small influence here, as Figures 3 and 4 make clear. Like the corresponding Figures 1 and 2 , both figures plot geometric mean magnitude estimates against SPL, Figure 3 giving the original data and Figure 4 the data after normalization. In both, I have continued to treat the subsets of SPLs within each set separately, as if here, too, as in Experiment 1, these subsets comprised different frequencies. And, in fact, the procedure of alternating between stimulus levels from the two subsets did produce some effect, with an average displacement between corresponding functions in Figure 4 equal to $6.1 \mathrm{~dB}$ (6.0 dB comparing first sessions only), or about $15 \%$ of the contextual shift in the stimulus levels. Thus, a 500-Hz tone can "mismatch" itself in loudness, the extent of mismatching (or, more properly, of change in matching values) being about $6 \mathrm{~dB}$ under present conditions of stimulation and judgment.

Without any qualitative distinction between the two sets of alternating stimuli-both sets comprised $500-\mathrm{Hz}$ tones-it seems unlikely that the subjects could have established independent frames of reference for them. More likely, the 6- $\mathrm{dB}$ shift in self-matches represents the "pure" effect of sequential biases in judgment; accordingly, by separating out the different subsets, I have plotted responses to stimuli as a function of the intensity levels presented on the immediately previous trial.

In Figures 3 and 4, open squares and filled triangles give results for low-SPL tones, but these immediately followed stimuli from the high-SPL set. Similarly, filled squares and open triangles give results for high-SPL tones, but these immediately followed stimuli from the low-SPL set. Because the low-SPL tones immediately followed stimuli from the high-SPL set, and vice versa, the fact that shifts appear in the judgments, and hence in "self matches," should not surprise, given the existence of sequential biases in judgment. In fact, the 6-dB shift is relatively modest in size, presumably because each plotted point entails averaging responses to a given stimulus following various SPLs from the other subset. Even greater shifts are evident if one examines responses to a given stimulus following every possible prior stimulus individually, as Cross (1973), for instance, has done.

Marks et al. (1986), following the work of Ward (1982), noted that sequential processes-in particular, the assimilation of each response to the response made to the prior stimulus-could account, in principle, for a sizable portion (about $50 \%$ ) of the bias they observed in cross- 


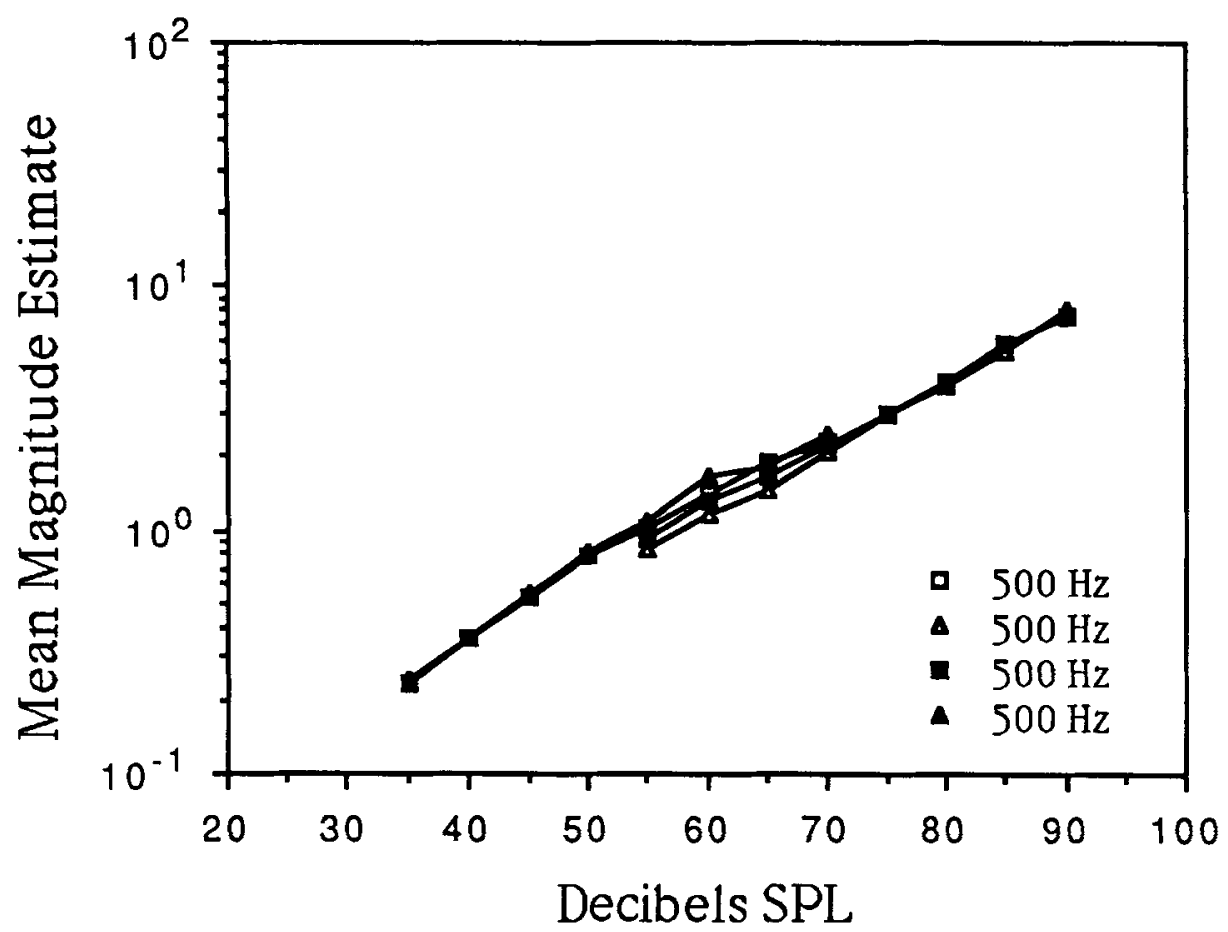

Figure 3. Geometric mean magnitude estimates of the loudness of $500-\mathrm{Hz}$ tones, plotted against sound pressure level (SPL). In the two conditions, subsets of tones were treated as if, as in Figure 1, they were qualitatively distinct: In Condition A (open symbols), stimulus presentations alternated between one subset of low SPLs (triangles) and another of high SPLs (squares), and in Condition B (filled symbols), in fact identical to $A$, between one subset of high SPLs (triangles) and another of low SPLs (squares).

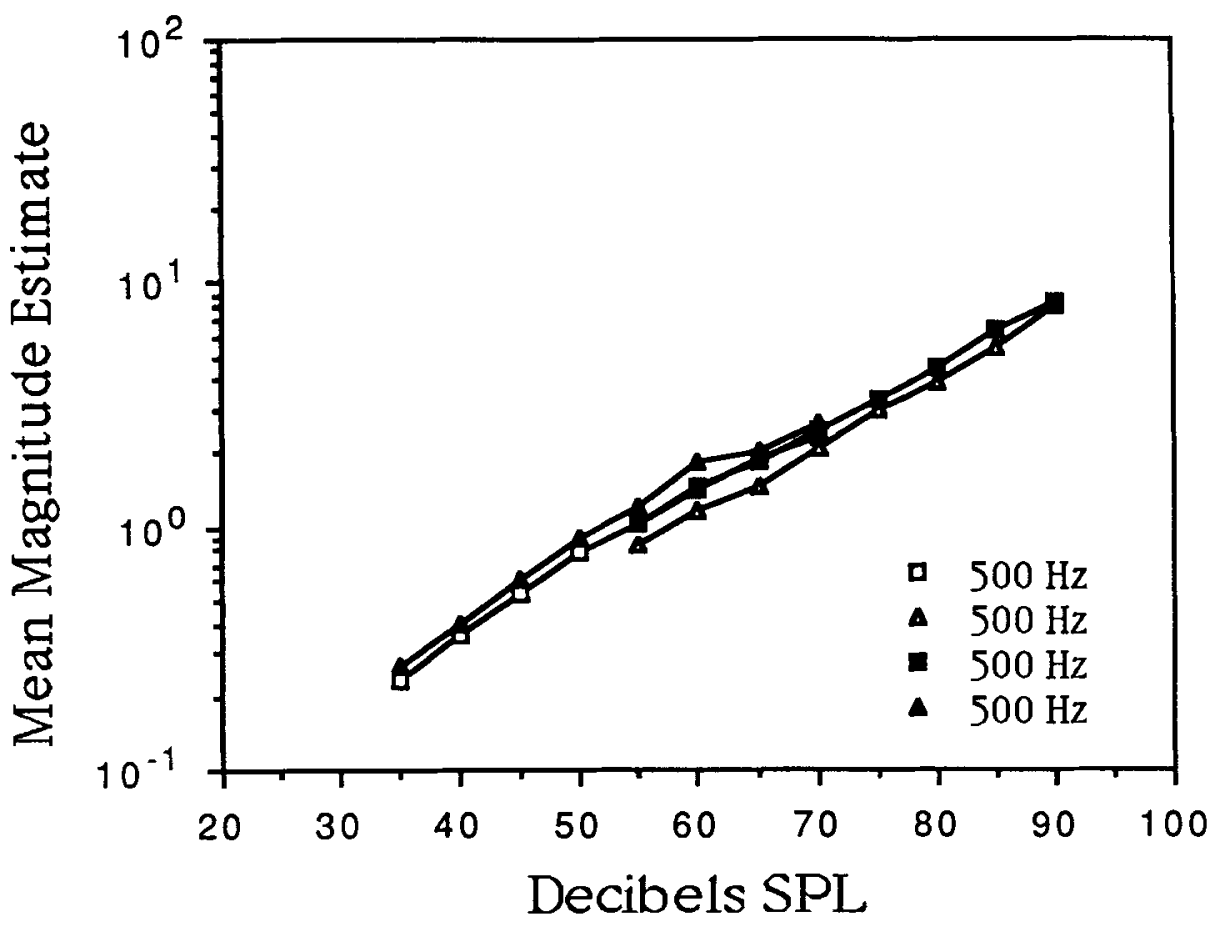

Figure 4. The data of Figure 3, normalized as in Figure 2. 
modal matches. It may be tempting to conclude from the present Experiments 1 and 2 that a similar sequential response assimilation operated in the present study, although accounting here for somewhat less than half of the intramodal bias (6.1 vs. $17.7 \mathrm{~dB}$ ). However, crossmodal and intramodal response assimilation need not be equivalent; indeed, assimilation itself could even vary with qualitative similarity-within as well as between modalities-perhaps being greatest in cross-modal comparisons, smaller in intramodal comparisons of qualitatively different stimuli, and smallest in intramodal comparisons of qualitatively identical stimuli. Thus, the results of Experiment 2 imply that sequential processes maybut need not-account for part of the shift in matches between tones of different frequency. In any case, the results of Experiment 2 are consistent with the general notion that context-induced bias is somehow related to qualitative similarity.

\section{EXPERIMENT 3}

The results of Experiment 2 suggest that the procedure of strictly alternating between subsets of the stimuli being compared is one source, presumably through shortterm sequential effects, of contextual bias. Alternation between stimulus subsets may have played at least a minor role in determining the results of Experiment 1, which compared $500-\mathrm{Hz}$ and $2500-\mathrm{Hz}$ tones. Experiment 3 sought to minimize, if not eliminate, this component by totally randomizing stimulus presentations of $500-\mathrm{Hz}$ and
$2500-\mathrm{Hz}$ tones so that every stimulus followed every stimulus, including itself, the same number of times. Although trial-to-trial sequential biases remain, randomization in principle should eliminate any systematic effect once data are averaged over the randomized trials. Thus, in each condition (A and B) of the present experiment, the average short-range sequential context was constant, and was equivalent for all stimuli.

\section{Method}

Experiment 3 reverted to the stimulus frequencies and SPLs of Experiment 1 . The only difference between Experiments 1 and 3 was in the construction of the stimulus orders: In each session of Experiment 3, the subject judged all 16 stimuli a total of 16 times each, with every stimulus following every other stimulus exactly once. The judgment of the stimulus presented on the first trial was not counted, since the first stimulus did not follow any other; instead, the first stimulus of each session was repeated at the session's end (trial number 257) to complete the balancing of firstorder sequential effects. Again, 16 young men and women participated; the stimulus order was different in each session for each subject.

\section{Results and Discussion}

Figures 5 and 6 display the results for original and normalized data, respectively. Somewhat surprisingly to me, the present results appear virtually identical to those of Experiment 1 . The change in matching stimulus values here is equal on average to $17.6 \mathrm{~dB}$, or $43 \%$ of the shift in stimulus context. (Note, though, that the change was $14.4 \mathrm{~dB}$ when first sessions only are compared, smaller

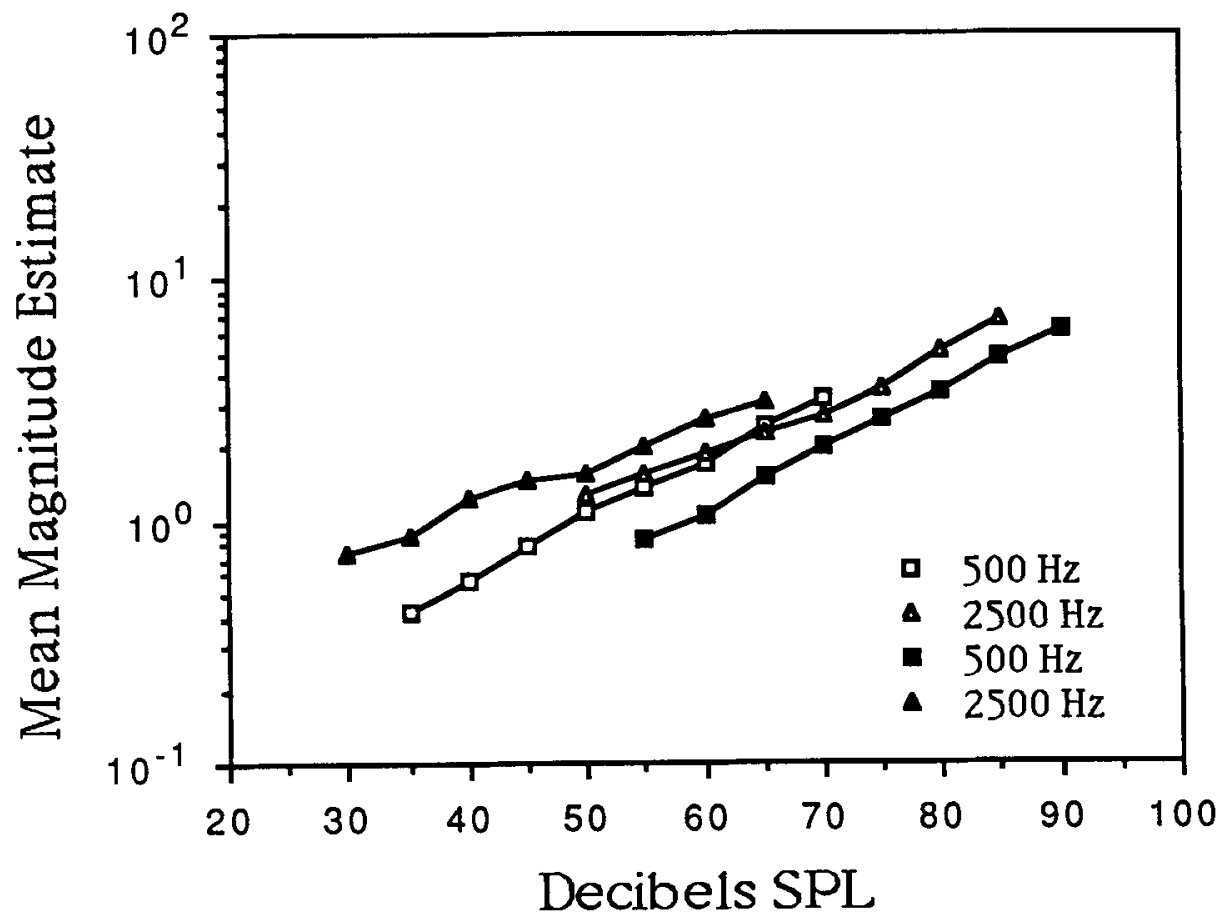

Figure 5. As in Figure 1, except that in each condition sequential effects were minimized by scheduling presentations within each session so that every stimulus followed every stimulus exactly once. 


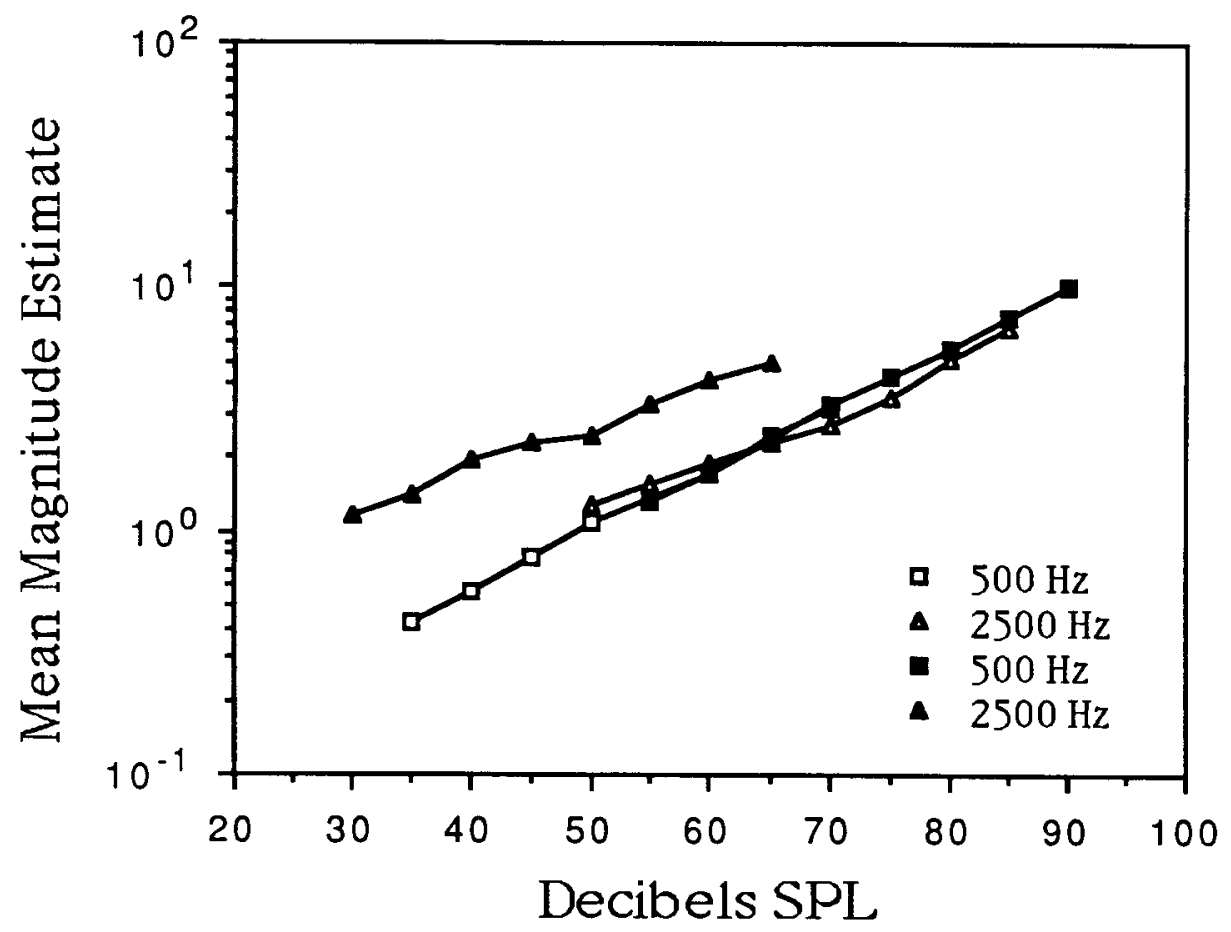

Figure 6. The data of Figure 3, normalized as in Figure 2.

than the corresponding value of $19.8 \mathrm{~dB}$ from Experiment 1.) The quantitative similarity between the results of Experiments 1 and 3 implies that first-order sequential effects-for instance, assimilation of the current response to the response on the previous trial-are secondary in importance, at least in intramodal comparisons of loudness. Even with total randomization of stimulus orders, and hence with neutralization of sequential biases, loudness matches between different sound frequencies, as derived from magnitude estimates, depend markedly and systematically on the set of stimulus levels being judged. Moreover, if it is appropriate to translate conclusions drawn from the present, intramodal, findings to the cross-modal case, then the results of Experiment 3 imply that Marks et al.'s (1986) suggestion-that sequential effects may account for about $50 \%$ of the contextual bias in cross-modal matches-is incorrect. The shifts in matching values have another, larger, source.

\section{EXPERIMENT 4}

Although heterofrequency loudness matches derived from magnitude estimates clearly can be "biased" by context, we do not yet know, of course, when or how such biases occur. This matter is both practical and theoretical in import. I have suggested-admittedly without much supporting data at this point-that bias occurs when the stimuli being compared are qualitatively different (as are tones of different frequency); by implication, I mean to suggest that other kinds of dissimilarity may not yield such bias (or may yield much less bias). I suspect, for exam- ple, much smaller biases would appear in loudness matches between auditory stimuli that differ in spatial location rather than in frequency, but that similarly large biases would appear in brightness matches between visual stimuli that differ in wavelength and that are presented under photopic conditions of viewing.

The success reported by J. C. Stevens and S. S. Stevens (1963), by Hellman and Zwislocki (1964), and by others lends support to the contention that magnitude estimates do provide accurate sensory matches when stimuli differ along dimensions other than quality. Stevens and Stevens compared the brightness aroused by white-light stimulation of a dark-adapted eye and of a white light-adapted eye; presumably, the various test stimuli varied greatly in brightness but only slightly in color. Hellman and Zwislocki compared the loudness of $1000-\mathrm{Hz}$ tones heard in quiet and in background noise; again, the test stimuli varied perceptually in intensity but not in quality. In both cases, matches derived indirectly, from the magnitude judgments, agreed with matches made directly.

It is conceivable, however, that in the aforementioned studies, scaling produced "good" matches because the levels and ranges of subjective magnitudes (the "contextual sets") were in rough accord; that is, in Stevens and Stevens's study, the lights viewed by the dark-adapted eye were about as bright as the lights viewed by the lightadapted eye, and in Hellman and Zwislocki's study, the tones in quiet were about as loud as the tones in noise. This explanation cannot account, however, for the findings of other studies. Both Raab (1962) and J. C. Stevens and Hall (1966) assessed the perception of brightness of 
flashes of white light that varied in both duration and luminance. Because brightness depends equally on duration and luminance over short durations (less than about $10 \mathrm{msec}$ ), in both studies the short-duration stimuli produced relatively low levels of brightness (that is, the levels of subjective magnitudes at different durations differed substantially). Nevertheless, both studies revealed a simple reciprocity between luminance and duration (Bloch's law), just like that obtained by direct matching (e.g., Aiba \& S. S. Stevens, 1964; Brindley, 1960). In sum, all of this evidence, albeit somewhat circumstantial, indicates that it is variation in qualitative similarity per se that is most important to context-induced shifts in sensory matches.

Experiment 4 provides a direct test. Using the method of Experiment 3, which wholly randomized stimulus orders and thus eliminated sequential effects as a source of bias in matches, I examined implicit loudness judgments of pure tones that appeared to be qualitatively identical, but that differed in apparent spatial location.

\section{Method}

The stimuli were 1-sec bursts of $500-\mathrm{Hz}$ tones, with $10-\mathrm{msec}$ rise and decay, presented to either the left or the right ear. In Condition A, the SPLs to the left ear were low (35-70 dB in 5-dB steps) and the SPLs to the right ear were high (55-90 dB); in Condition B, the stimulus levels were reversed (high to the right ear and low to the left). As in Experiment 3, each of the 16 subjects judged all 16 stimuli of a given condition a total of 16 times each in the course of the session (plus the first stimulus of the session repeated at the end as trial 257); within the session, every stimulus followed every other stimulus once.

\section{Results and Discussion}

Original and normalized data appear in Figures 7 and 8 , respectively. Although the data do reveal a small effect of changing the sets of stimulus levels being judgedas Figure 7 indicates, in each condition the judgments of low SPLs are a bit higher than the corresponding judgments of high SPLs-the contextual bias is much more modest than that obtained in either Experiment 1 or Experiment 3 . The shift in matching levels here equals only $5.95 \mathrm{~dB}$; this value, given the $40-\mathrm{dB}$ change imposed on the stimulus sets, corresponds to a contextual matching bias of $14.9 \%$. Clearly, the subjects found it relatively much easier to maintain loudness matches in the face of this nonqualitative difference between sensory experiences-a difference in spatial location-than they did in the face of a large qualitative difference.

It is possible, of course, that the magnitude of contextinduced bias depends on some measure of overall psychological difference or dissimilarity, and that the difference between left and right ears is simply smaller than the difference between $500 \mathrm{~Hz}$ and $2500 \mathrm{~Hz}$. But I suspect instead that the central factor is nonqualitative versus qualitative dissimilarity. By this token, I would expect greater bias when the stimuli being compared differ in quality than when they differ in some other way, even if the differences in dissimilarity are equal. Therefore,

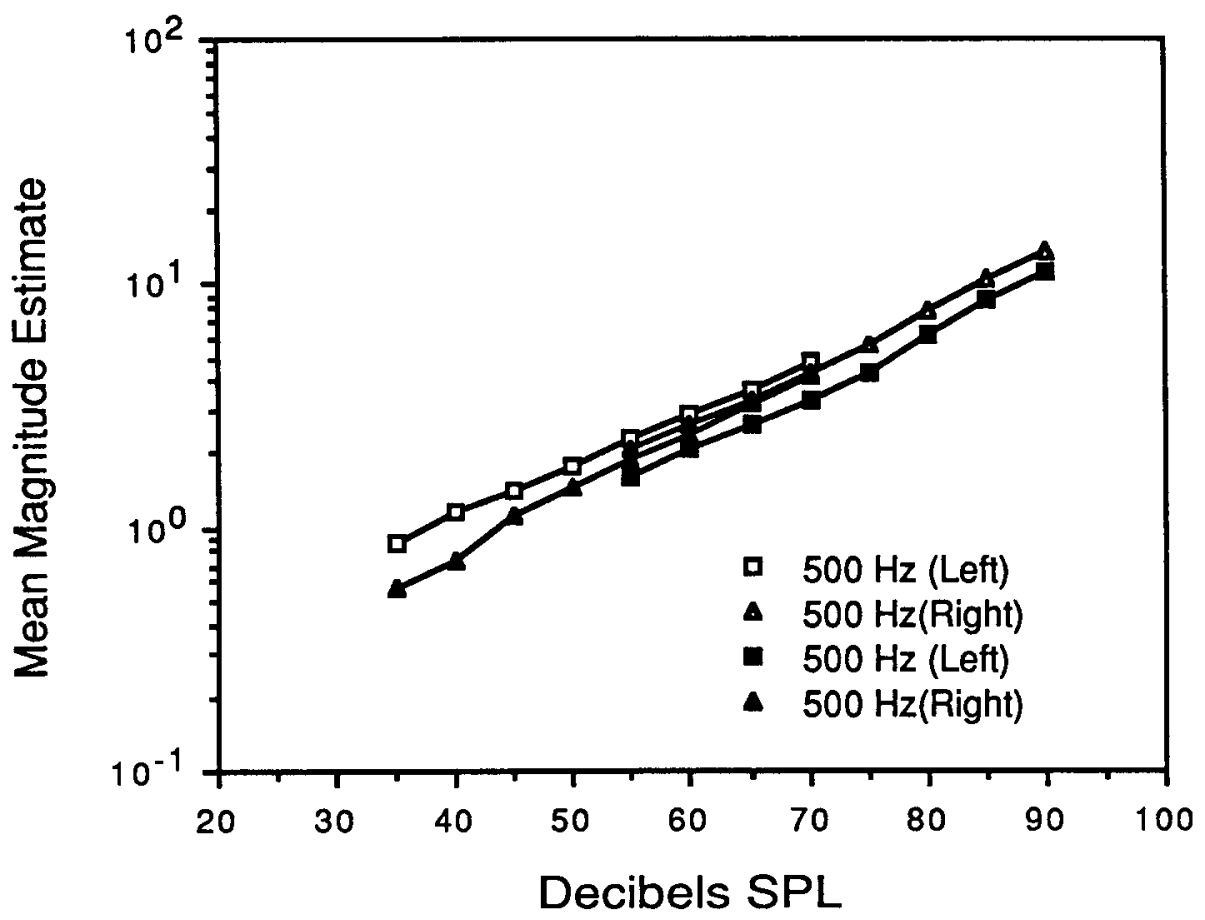

Figure 7. Geometric mean magnitude estimates of loudness of $500-\mathrm{Hz}$ tones presented to the left ear (squares) or to the right ear (triangles), plotted against sound pressure level (SPL). In each condition (A and B), every stimulus followed every stimulus exactly once. In Condition A, the SPLs to the left ear were low and those to the right were high (open symbols); in Condition B, those to the left ear were high and to the right, low (filled symbols). 


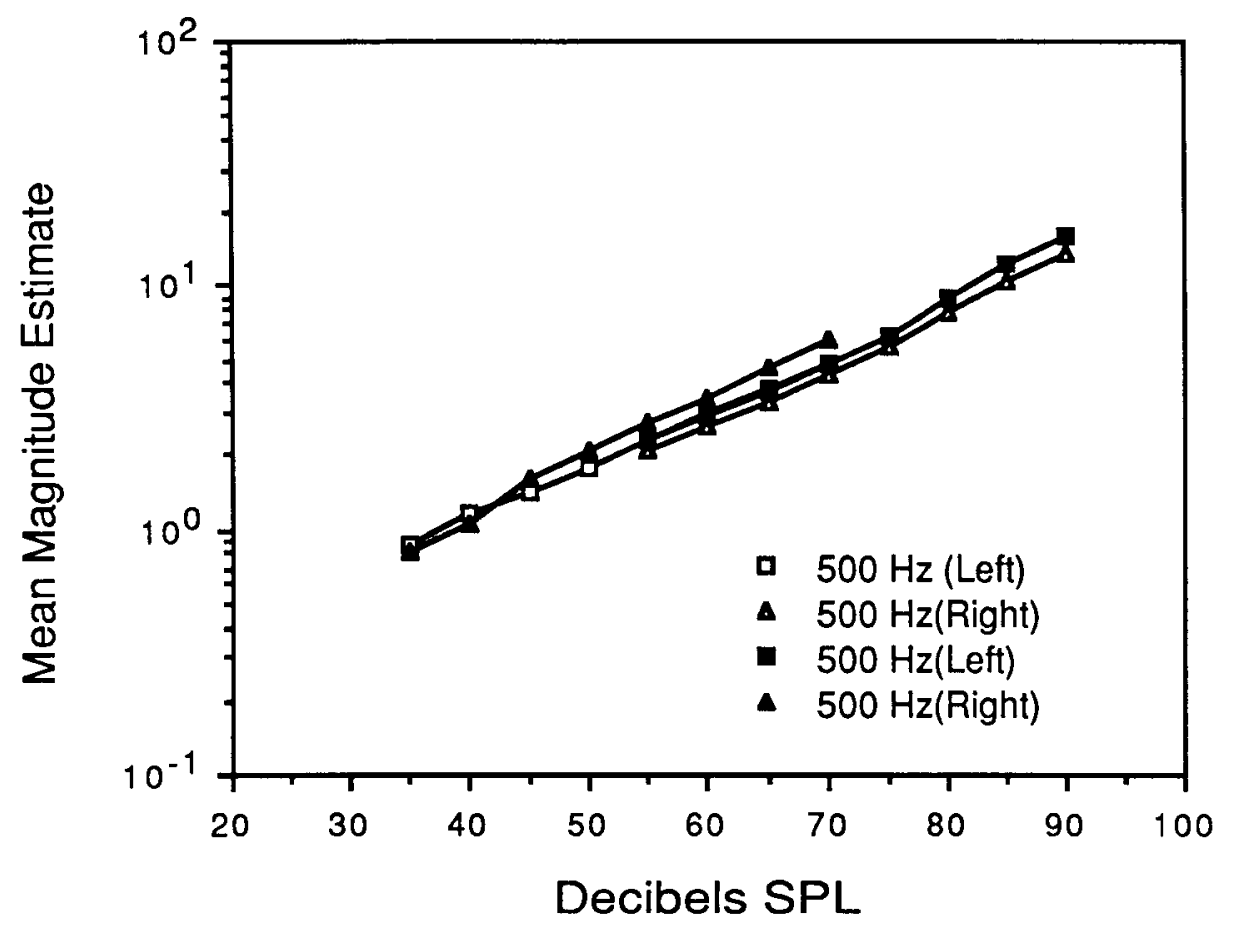

Figure 8. The data of Figure 7, normalized as in Figure 2.

the present results are consistent with the contention that the data obtained from scaling experiments such as those of Hellman and Zwislocki (1964), Raab (1962), J. C. Stevens and Hall (1966), and J. C. Stevens and S. S. Stevens (1963), which required subjects to judge stimuli that varied on nonqualitative dimensions, yielded "good" matches, in that the matching stimulus values are not highly susceptible to "contextual bias."

\section{EXPERIMENT 5}

The results of Experiments 1-4 reveal the existence of contextual biases that can markedly influence loudness matches derived from numerical judgments given to qualitatively different acoustic stimuli. In all of those experiments, in order to vary the context from condition to condition, I shifted the SPLs at a given frequency up or down by $20 \mathrm{~dB}$. This maneuver produced a $20-\mathrm{dB}$ increase or decrease in the average SPL and a corresponding $20-\mathrm{dB}$ increase or decrease in the lowest and highest SPLs of the stimulus set. But which factor determines the contextual bias in matches? Or do both factors do so? Do matches shift when the average stimulus (or perceptual) magnitude changes? Or do they do so when the intensity levels that constitute the top and bottom change?

Experiment 5 sought to help answer this question by holding the stimulus levels constant over sessions, and therefore leaving the lowest and highest SPLs unchanged, but varying the proportion of times each stimulus level was presented for judgment, and thereby varying the average stimulus magnitude over conditions. The method of varying the distribution of presentations has often been used to study how context affects categorical judgments (e.g., Parducci, 1965; Parducci \& Perrett, 1971). The evidence from those studies-that subjects are sensitive to the distribution of stimulus or sensory events over the course of a session-suggests that variations in stimulus distribution alone, like variations in absolute stimulus levels, might also affect implicit loudness matches between $500-\mathrm{Hz}$ and $2500-\mathrm{Hz}$ tones.

\section{Method}

Unlike the previous experiments of this study, the SPLs in Experiment 5 remained constant across the two conditions; in both conditions, there were nine SPLs at $500 \mathrm{~Hz}(45-85 \mathrm{~dB}$ in 5-dB steps) and nine SPLs at $2500 \mathrm{~Hz}(40-80 \mathrm{~dB}$ in 5-dB steps). However, the distributions of stimulus presentations differed across conditions. In Condition A, most of the presentations at $500 \mathrm{~Hz}$ were at the low SPLs; the nine SPLs were presented according to the frequency distribution 10, 4, 3, 2, 2, 1, 1, 1, and 1, from low to high. Most of the presentations at $2500 \mathrm{~Hz}$ were high; the nine SPLs were presented according to the reverse distribution. Given these distributions, the weighted mean SPL at $500 \mathrm{~Hz}$ was $55 \mathrm{~dB}$, and that at $2500 \mathrm{~Hz}$ was $70 \mathrm{~dB}$. In Condition $\mathrm{B}$, the frequency distributions were switched, so most of the presentations at $500 \mathrm{~Hz}$ were now high, with a mean SPL of $75 \mathrm{~dB}$, and most of those at $2500 \mathrm{~Hz}$ were now low, with a mean SPL of $50 \mathrm{~dB}$. Note that at each sound frequency, the mean SPL shifted by $20 \mathrm{~dB}$ across conditions, just as each did in previous experiments. But the present $20-\mathrm{dB}$ shifts across conditions were implemented without changing the SPLs themselves.

A single replicate consisted of 50 trials (the 25 presentations at each sound frequency for the condition) with the stimuli given in random order. Four replicates constituted a given session for each of the 16 subjects, half of whom participated first in Condition A and subsequently in Condition $\mathrm{B}$. The sequence was reversed for the remaining subjects. 


\section{Results and Discussion}

The average magnitude estimates are plotted in Figure 9; Figure 10 plots the data normalized so that the functions at $500 \mathrm{~Hz}$ overlap. It is clear that manipulating the distribution of stimulus presentations had a marked differential influence on the judgments given to the two sound frequencies, and hence on the implied loudness matches. The shift between conditions amounts to $11.3 \mathrm{~dB}$, a substantial amount, although only about twothirds the magnitude of the shift observed in Experiment 3, in which I manipulated the intensity levels themselves. Clearly what matters to sensory equivalence is not only the intensities that define the endpoints of the distributions, but also the distributions themselves and perhaps some measure of an average value.

Parducci has studied how the distribution of stimuli affects categorical judgments, and he has developed a rangefrequency model (Parducci, 1965; Parducci \& Perrett, 1971) to account for subjects' tendency to assign a constant number of stimulus presentations to each response category. In elaborating this model, Parducci, Knobel, and Thomas (1976) suggested that the psychological endpoint moves farther out when stimuli from that end of the range are presented more often; thus it may be that skew-

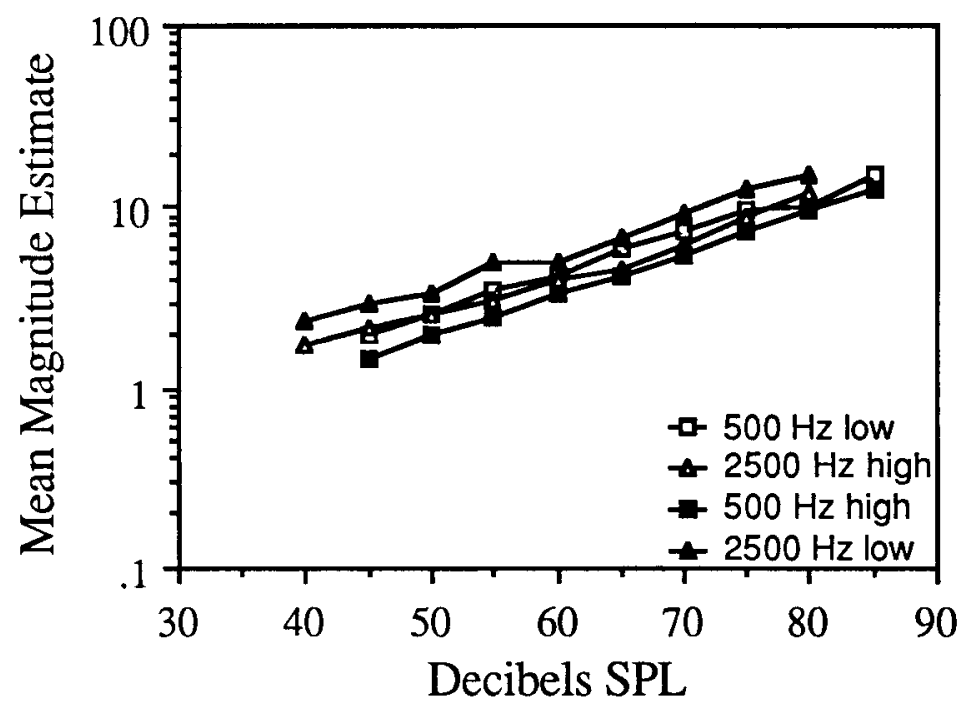

Figure 9. Geometric mean magnitude estimates of the loudness of $500-\mathrm{Hz}$ tones (squares) and 2500-Hz tones (triangles), plotted against sound pressure level (SPL). In Condition A, most of the intensity levels presented at $500 \mathrm{~Hz}$ were low, and most of those presented at $2500 \mathrm{~Hz}$ were high (open symbols); in Condition $B$, most of the intensity levels presented at $500 \mathrm{~Hz}$ were high, and most of those presented at $2500 \mathrm{~Hz}$ were low (filled symbols).

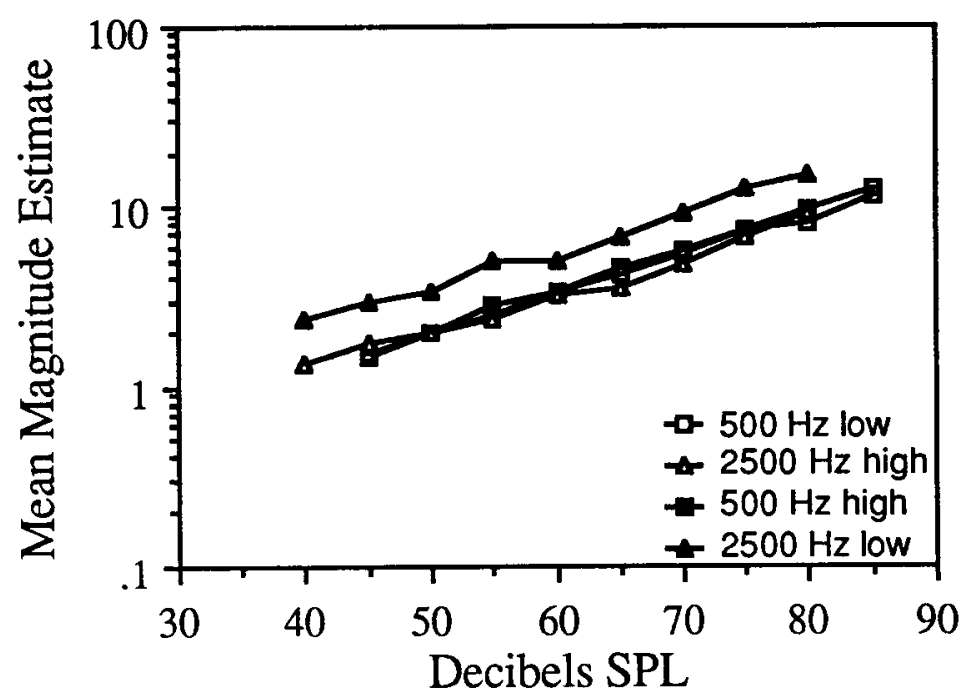

Figure 10. The data of Figure 9, normalized as in Figure 2. 
ing the stimulus distribution does function psychologically much like shifting stimulus levels themselves.

Although much of Parducci's work has focused on judgments of single stimulus sets, he has also asked how well subjects can maintain independent contexts when judging two sets (Parducci et al., 1976). Subjects were instructed to rate the sizes of squares and circles presented on alternate trials within the same session. In different conditions, the distributions of the circle sizes and of the square sizes varied. In every condition, all of the circles were larger than all of the squares; but the subjects were instructed to judge each set independently, that is, to use the category range from "very very small" to "very very large" separately for each set. Such instructions in essence imply that subjects need not be concerned with matches between stimuli of different shapes. Three results are noteworthy. (1) The distribution of stimulus values in any set-circles or squares-affected the ratings given to that set, but had no effect on the ratings given to the other set. That is, the changes in context (i.e., in stimulus distribution) exerted independent effects. (2) However, the ratings given to the circles (all physically larger than the largest square) were somewhat higher than those given to squares, although there was considerable overlap in response range. That is, the judgments made to circles and to squares were partially linked, presumably because of the absolute stimulus levels. So although changes in the two stimulus distributions produced independent effects, the different absolute stimulus magnitudes in the two sets did interact. (3) Perhaps most directly relevant to the present study was the finding obtained in a control condition. When the subjects were instructed to judge the circles and squares on a single scale, changes in stimulus distributions did have an effect. From the perspective of size matches, the matching values between the two sets of stimuli were compromised by changes in the stimulus distributions. In sum, these findings suggest that the absolute perceptual magnitudes matter even when subjects try to ignore them and apply contexts independently, and that contexts matter even when subjects try to ignore them and judge perceptual magnitudes dependently.

In an unpublished study, Riskey and Desor (1980) observed similar interactions in category judgments of taste intensity of sucrose and sodium chloride; their results imply a shift in matching values of taste intensity with changes in stimulus distribution, much like those of the present experiment. Both the results of Parducci et al. (1976) and those of Riskey and Desor support the view that matches can shift when there is qualitative dissimilarity. More recently, Vollmecke (1987) found that when subjects judged the sweetness of varying concentrations of sucrose in beverages containing different flavorings (apricot and papaya), the subjects did succeed in evaluating all of the sensations on a uniform scale. Just as Experiment 4 showed interaural matches of quantitatively identical tones to be largely independent of context, Vollmecke showed that changing context (distribution) of sucrose concentrations in different flavorings did not affect sweetness matches (although context did affect pleasantness matches, as Riskey and Desor also found). So for judgments of intensity, at least, the factor crucial to whether matches are reasonably invariant may be the identity or nonidentity of the quality whose intensity is judged.

\section{GENERAL DISCUSSION}

These results are intriguing and, despite the modulating effect of the last experiment, profoundly disturbing. Not only can contextual biases affect scaling behavior (e.g., Garner, 1954; Helson, 1964) and cross-modal comparisons (Marks et al., 1986; Melamed, 1970; K. Smith \& Hardy, 1961), but context can strongly disrupt matches within a modality. Thus, these results have both practical and theoretical consequences.

First, a practical one: The present findings suggest that in using scaling methods to evaluate sensory processes, and in particular to generate intensity matches, care should be taken in choosing stimulus levels. Ideally, to ensure that derived intensity matches are accurate, the levels and the distributions of sensory magnitudes should be the same for all qualitatively different stimuli being judged. This conclusion rests on two tacit assumptions. First, I assume that the changes in matching values observed with shifts in stimulus context do involve some kind of "assimilation" of judgments (even if the assimilation is not simply the result of sequential processes in judgment). Second, and consequently, I assume that assimilation is negligible when the levels or perhaps the means of the sensory values on the two sets of stimuli being compared are identical. Of course, equalizing sensations entails knowing before the experiment is undertaken precisely what it is that the experiment aims at identifying-namely, the matching sensory values. Preliminary experimentation can often provide at least a rough approximation. A more thorough and systematic approach is to iterate experiments (a procedure that, for related purposes, has been recommended to eliminate biases in rating scales: see Pollack, 1965; S. S. Stevens \& Galanter, 1957); because the contextually based shifts are only partial, successive experimental approximations can yield increasingly precise matching values.

The practical consequences also speak to the question of scaling method. All of the experiments reported here used magnitude estimation, largely because magnitude estimation appears to be the method most often employed when investigators use scaling methods to assess sensory functioning. Other scaling methods-for instance, category or graphic rating-doubtless will show similar degrees of contextual bias, as results of Parducci et al. (1976) and Riskey and Desor (1980) indicate. Moreover, in cross-modal comparison of loudness and taste intensity, graphic rating and magnitude estimation revealed similarly large changes in sensory matches under the challenge of shifting context (Marks et al., 1988). 
An overarching theoretical issue is: What is the source of the contextual bias? It seems not to depend to any great extent on sequential processes, although these may play a minor role. To describe the contextual bias in terms of changes in "adaptation level" is not helpful until we can identify the factors that control such changes. Although we don't yet know enough about the sources of contextual bias to construct a detailed, formal model, we can take some preliminary steps.

\section{Models of Matching "Bias"}

One simple set of descriptive models builds on the wellknown phenomenon variously called the "central tendency of judgment" (Hollingworth, 1916) or "regression" (S. S. Stevens, 1975) - the tendency for people to moderate extreme judgments or restrict the range of responses. Figure 11 depicts three increasingly more complex applications of centering or regression to judgments of a series of intensity levels of two stimuli that vary in perceived quality as well as in perceived intensity. For convenience (although this is not necessary to the argument), perceived

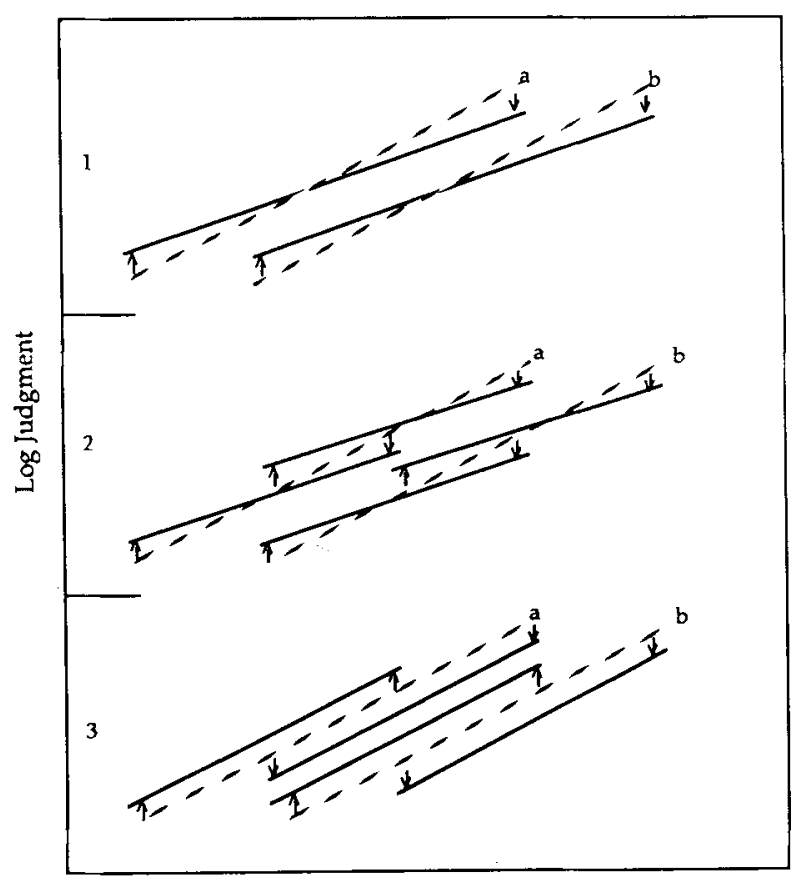

Log Stimulus

Figure 11. Three models based on processes of "centering," which transform underlying perceived magnitudes (dashed lines) into numerical responses (solid lines). In Model 1 (top), the degree of centering of qualitatively different stimuli, $a$ and $b$, is independent of quality and depends solely on underlying perceived magnitude; the matching values between $a$ and $b$ do not change. In Model 2 (middle), each subset of intensities of each quality undergoes its own centering; matches between stimuli from the low-intensity subset of $a$ and the high-intensity subset of $b$ differ from matches between stimuli from the high-intensity subset of $a$ and the low-intensity subset of b. In Model 3 (bottom), responses to the subset of each quality are assimilated to the levels of the other quality; again, matches shift across contextual conditions. intensity is represented as a power function of stimulus intensity.

In its simplest version, centering or regression would apply equally to all qualitatively different stimuli, its magnitude being dependent solely on overall mean perceived intensity. A general equation for Model 1 states that

$$
R=T(\log I ; \log I),
$$

where $R$ is the $\log$ response after centering, and where the centering transformation $T$ operates on the perceived intensity of the stimulus $(\log I)$ and on some measure of average perceived intensity $(\log I)$. As represented in the upper portion of Figure 11, centering acts on perceived intensities, given by the dashed lines, to produce responses, given by the solid lines; because the degree of centering depends only on the level of perceived intensity (the value on the $y$-axis), matching values are unaffected. All three models depicted in Figure 11 assume $T$ to be a power transformation in which

$$
R=\log I+c(\overline{\log I}-\log I) .
$$

Model 1 is essentially the one that underlies the principle of "matching-by-scaling" (e.g., Marks, 1974). As we have seen, the present data strongly reject the adequacy of this model when applied to loudness judgments made to tones of different frequencies, although it may apply better to judgments made to sensations that do not differ qualitatively.

A more complex version of the model assumes that centering applies separately to each qualitatively distinct subset of stimuli (middle part of Figure 11). Therefore, as the judgments given to a low-intensity subset of stimuli of quality $a$ undergo their centering, those given to a highintensity subset of stimuli of another quality $b$ undergo theirs. When, by experimental manipulation, the subsets change differentially, the net result, according to Model 2 and as shown in the middle section of Figure 11, is a shift in matching stimulus values in the predicted direction. For example, a small log stimulus difference separates the lowintensity subset of $a$ and the high-intensity subset of $b$, but a considerably larger stimulus difference separates the high subset of $a$ from the low subset of $b$.

Three detailed considerations of this model indicate, however, that it too is inadequate to account for the present results. First, Model 2 implies that the size of the shift in matching values is directly related to the value of the centering parameter $c$ in Equation 2, which in turn directly affects the log-log slope of the psychophysical function. By linking the slope of the function to the degree of bias, the model would lead us to expect similar degrees of matching bias in Experiments 1, 3, and 4, given the similar experimental manipulations. Moreover, given that the contextually based shifts run about $15-40 \%$, we would have to assume that the underlying psychophysical relations between loudness and sound pressure "precentering" are about $30-80 \%$ greater in slope (power-function exponent) than those obtained. This does not seem reasonable. Finally, although the heterofrequency matches 
do change in the predicted direction, the psychophysical functions do not. The dashed-line (precentering) functions in Figure 11 were generated on the assumption that the subjective levels of the two stimuli were about equal (as, presumably, was roughly the case in all of the experiments). Given equal subjective levels, centering should result, according to Model 2, in the high-intensity subset of each stimulus perching above the corresponding lowintensity subset. But the data (e.g., Figures 1 and 5) show just the reverse: The low-intensity subsets perch above the high-intensity subsets.

Model 3, depicted in the lower portion of Figure 11, makes predictions that correspond more closely to this outcome. According to this model (which, though an assimilation model, perhaps technically should not be considered a centering model), the judgments made to each qualitatively distinct subset of stimuli are shifted in the direction of the average perceived magnitude of the other qualitative subset. Hence, the responses to low-intensity levels of quality $a$ shift toward high ones of quality $b$, and high levels toward low ones. Model 3 is described by the equations

$$
\begin{aligned}
& R_{a}=\log I_{a}+c_{a b}\left(\overline{\log I_{b}}-\log I_{a}\right), \\
& R_{b}=\log I_{b}+c_{b a}\left(\overline{\log I_{a}}-\log I_{b}\right),
\end{aligned}
$$

where the values of $c_{a b}$ and $c_{b a}$ represent the magnitude of the shift in judgment, and may reflect the degree of qualitative similarity. Centering tendencies corresponding to Model 1 may also take place, but these are not observed directly in terms of changes in sensory matches. Be this as it may, Model 3 gives a qualitatively adequate account of the findings.

\section{Encoding Sensory Intensity}

Although it is feasible to derive a descriptive, quantitative model to describe the way loudness judgments change with changes in subsets of stimulus levels, a deeper theoretical issue concerns the way loudness is encoded. Two not necessarily incompatible interpretations come to mind. One states that, to use Garner's (1974) terminology, loudness is not wholly separable from other perceived attributes and dimensions, especially pitch. Wood (1975) found interactions between loudness and pitch when measuring response speed and accuracy in an identification task. Perhaps there is a continuum of integrality-separability vis-à-vis loudness and other attributes (cf. L. B. Smith \& Kilroy, 1979); if so, then if I were to use the magnitude of the shift in loudness matches as a measure of separability, I would expect to find the degree of separability to vary systematically, but inversely, with the degree of qualitative dissimilarity (e.g., with difference in frequency of pure tone, with tone vs. noise, and so on) and, perhaps to a smaller extent, with other types of dissimilarity (e.g., with apparent spatial location, duration). The present results imply that the dissimilarity between tones presented to different ears is not so great as the dissimilarity between $500-\mathrm{Hz}$ and $2500-\mathrm{Hz}$ tones.
At this point, it is important to be careful in using the term "separable." It is not clear, for instance, how the present characterization of "separability" would compare to that derived from more traditional measures, such as independence of response speed in sorting tasks, although Wood's (1975) results suggest that the two characterizations may be related. Moreover, we may inquire whether analogous results would obtain if the variables in the scaling experiment were reversed-that is, if subjects judged the pitch of tones varying in frequency when the tones were presented at two levels of loudness and the subsets of frequencies were varied over conditions. Here, I suspect that changing context would exert a relatively small effect. If this is so, then we might be forced to conclude that separability of loudness and pitch is asymmetrical. Garner (e.g., 1974) has discussed instances of asymmetrical interactions between dimensions.

Another conceptual scheme, which is wholly consistent with the aforementioned notion of "separability," centers on a notion of "channels." According to this scheme, a single sound-even a pure tone-generates a loudness that is encoded as values on a set of overlapping (broadly tuned) channels; the channels do not vary greatly with changes in such nonqualitative parameters as duration or spatiality, but are characterized largely in terms of some function of sound frequency. Perhaps the unit is the critical bandwidth. One analogue appears in the notion of "specific loudness" - the theoretical contribution to loudness made by each critical band, according to the model of Zwicker and Scharf (1965). Another analogue appears at a neurophysiological level, in which a tone of fixed frequency and SPL may primarily stimulate neural units whose characteristic frequency lies close to the frequency of the test tone, although it will also stimulate units that have neighboring characteristic frequencies (Kiang, Watanabe, Thomas, \& Clark, 1965). Comparisons of loudness between different stimuli are most precise when the stimuli arouse the same distribution of activity in the frequency channels (Brindley, 1960, Class A)-for instance, when the stimuli comprise pure tones with identical sound frequency (e.g., Robinson \& Dadson, 1956; see Lim, Rabinowitz, Braida, \& Durlach, 1977 , for a model of heterofrequency loudness comparison that employs a parameter to account for dissimilarity based on frequency difference).

When different stimuli arouse the same distribution of activity in the channels, not only are direct matches most precise, but indirect matches derived from scaling judgments-tasks requiring greater resources of memory-are most absolute. Furthermore, I assume that in the absence of any overlap in channels, matches derived indirectly would be maximally relative. The degree of "absoluteness" should correspond to the degree of overlap. The results of the present Experiments 1 and 3 imply that frequency separations of several critical bandwidths $(500 \mathrm{~Hz}$ and $2500 \mathrm{~Hz}$ are separated by about $8-9$ critical bandwidths) still permit about $50-60 \%$ communality among channels. Moreover, the results of Marks 
et al. (1986) and Marks et al. (1988) on cross-modal judgments of intensity suggest that a similar value-about $50 \%$ communality - characterizes comparisons between loudness and brightness, between loudness and vibration, and between loudness and taste. From the present point of view, then, different modalities function much like widely separated sound frequencies. Still lacking are both a thorough assessment of how contextual shifts in matches depend on qualitative and other kinds of similarity in various sense departments, and a clear linkage between a qualitative hypothesis, such as the characterization of channels just outlined, and a quantitative model.

\section{REFERENCES}

Aiba, T. S., \& Stevens, S. S. (1964). Relation of brightness to duration and luminance under light- and dark-adaptation. Vision Research, 4, 391-401.

BrindLey, G. S. (1960). Physiology of the retina and visual pathway. London: Arnold.

CoHEN, N. E. (1934). Equivalence of brightnesses across modalities. American Journal of Psychology, 46, 117-119.

Cross, D. V. (1973). Sequential dependencies and regression in psychophysical judgments. Perception \& Psychophysics, 14, 547-552.

Fletcher, H., \& Munson, W. A. (1933). Loudness, its definition, measurement and calculation. Journal of the Acoustical Society of America, 5, 82-108.

GarNER, W. R. (1954). Context effects and the validity of loudness scales. Journal of Experimental Psychology, 48, 218-224.

GARNER, W. R. (1974). The processing of information and structure Potomac, MD: Erlbaum.

Hellman, R. P., Zwislocki, J. J. (1964). Loudness function of a 1000-cps tone in the presence of a masking noise. Journal of the Acoustical Society of America, 36, 1618-1627.

Helson, H. (1964). Adaptation-level theory: An experimental and systematic approach to behavior. New York: Harper \& Row.

HoluingwORTH, H. L. (1916). The central tendency of judgment. Journal of Philosophy, Psychology, \& Scientific Methods, 7, 461-469.

HowEs, D. H. (1950). The loudness of multicomponent tones. American Journal of Psychology, 63, 1-30.

Jesteadt, W., Luce, R. D., \& Green, D. M. (1977). Sequential effects in judgments of loudness. Journal of Experimental Psychology: Human Perception \& Performance, 3, 92-104.

Kiang, N. Y.-S., Watanabe, T., Thomas, E. C., \& Clark, L. F. (1965). Discharge patterns of single fibers in the cat's auditory nerve. Cambridge, MA: MIT Press.

Lim, L. S., Rabinowitz, W. M., Braida, L. D., \& Durlach, N. I. (1977). Intensity perception: VIII. Loudness comparisons between different types of stimuli. Journal of the Acoustical Society of America, 62, 1256-1267.

LOCKHEAD, G. R., \& KING, M. C. (1983). A memory model of sequential effects in scaling tasks. Journal of Experimental Psychology: Human Perception \& Performance, 9, 461-473.

MARKs, L. E. (1974). Sensory processes: The new psychophysics. New York: Academic Press.

MARKS, L. E. (1979). A theory of loudness and loudness judgments. Psychological Review, 86, 256-285.
Marks, L. E., Stevens, J. C., Bartoshuk, L. M., Gent, J. F., RifKIN, B., \& Stone, V. K. (1988). Magnitude matching: The measurement of taste and smell. Chemical Senses, 13, 63-87.

Marks, L. E., Szczesiul, R., \& Ohlott, P. (1986). On the crossmodal perception of intensity. Joumal of Experimental Psychology: Human Perception \& Performance, 12, 517-534.

MELAMED, L. E. (1970). The role of response processes in the formation of cross-modality assimilation effects. Perception \& Psychophysics, 8, 185-188.

Mellers, B. A., \& Birnbaum, M. H. (1982). Loci of contextual effects in judgment. Journal of Experimental Psychology: Human Perception \& Performance, 8, 582-601.

ParduCCI, A. (1965). Category judgment: A range-frequency model. Psychological Review, 72, 407-418.

Parducci, A., Knobel, S., \& Thomas, C. (1976). Independent contexts for category ratings: A range-frequency analysis. Perception \& Psychophysics, 20, 360-366.

Parducci, A., Perrett, L. F. (1971). Category rating scales: Effects of relative spacing and frequency of stimulus values. Journal of Experimental Psychology Monographs, 89, 427-452.

Pollack, I. (1965). Iterative techniques for unbiased rating scales. Quarterly Journal of Experimental Psychology, 17, 139-148.

RAAB, D. H. (1962). Magnitude estimation of the brightness of brief foveal stimuli. Science, 135, 42-44.

RISKEY, D., \& DESOR, J. (1980). Independence and nonindependence between taste contexts. Unpublished manuscript.

Robinson, D. W., \& Dadson, R. S. (1956). A re-determination of the equal-loudness relations for pure tones. British Journal of Applied Physics, 7, 166-181.

Smith, K., \& HardY, A. H. (1961). Effects of context on the subjective equation of auditory and visual intensities. Science, 134, 1623-1624.

SMITH, L. B., \& KILRoY, M. C. (1979). A continuum of dimensional separability. Perception \& Psychophysics, 25, 285-291.

Stevens, J. C., * HaLL, J. W. (1966). Brightness and loudness as functions of stimulus duration. Perception \& Psychophysics, 1, 319-327.

Stevens, J. C., \& MARKs, L. E. (1980). Cross-modality matching functions generated by the method of magnitude estimation. Perception \& Psychophysics, 27, 379-389.

Stevens, J. C., \& STevens, S. S. (1963). Brightness function: Effects of adaptation. Joumal of the Optical Society of America, 53, 375-385.

Stevens, S. S. (1975). Psychophysics: Introduction to its perceptual, neural, and social prospects. New York: Wiley.

STEVENS, S. S., \& GaLANTER, E. (1957). Ratio scales and category scales for a dozen perceptual continua. Journal of Experimental Psychology, 54, 377-411.

VOLLMECKE, T. A. (1987). The influence of context on sweetness and pleasantness evaluation of beverages. Unpublished doctoral dissertation, University of Pennsylvania. Philadelphia.

WARD, L. M. (1973). Repeated magnitude estimations with a variable standard: Sequential effects and other properties. Perception \& Psychophysics, 13, 193-200.

WARD, L. M. (1982). Mixed-modality psychophysical scaling: Sequential effects and other properties. Perception \& Psychophysics, 31, 53-62.

WooD, C. C. (1975). Auditory and phonetic levels of processing in speech perception: Neurophysiological and information-processing analyses. Journal of Experimental Psychology: Human Perception \& Performance, 1, 3-20.

ZWICKER, E., SCHARF, B. (1965). A model of loudness summation. Psychological Review, 72, 3-26. 